\title{
Application Value of Vitamin C in the Treatment of Sepsis
}

\author{
Qiong Hang* \\ Xinrui Hospital, Wuxi 214000, Jiangsu Province, China \\ *Corresponding author: Qiong Hang, 124168479@qq.com
}

\begin{abstract}
Objective: To analyze the effect and value of vitamin C in the treatment of sepsis. Methods: Forty-four patients with sepsis treated in our hospital from June 2019 to June 2021 were investigated. The above subjects were divided into conventional group and research group, each with 22 cases, using the method of drawing lots. Routine treatment was applied in the conventional group, and the patients of the research group were treated with vitamin $\mathrm{C}$ on the basis of the routine method. The effective rate after treatment and the score of liver and kidney function in patients with sepsis were compared between the two groups. Results: The total effective rate (95.45\%) of the research group with increased vitamin C in the treatment of sepsis was much higher than that of the conventional group $(72.72 \%)$, and the scores of liver function and renal function in the research group were much higher than those in the conventional group at different times $(\mathrm{P}<0.05)$. Conclusion: Vitamin $\mathrm{C}$ has a good effect in the treatment of sepsis. It not only greatly reduces the mortality of sepsis patients, but also effectively improves the liver function and renal function of sepsis patients. It is of high application value. It is recommended to be popularized for applications.
\end{abstract}

Keywords: Vitamin C; Sepsis; Treatment; Application value

Publication date: November 2021; Online publication: November 30, 2021

\section{Introduction}

Sepsis refers to the life-threatening organ dysfunction caused by the maladjusted response of the body to infection. This disease is accompanied by acute onset. Patients with sepsis will suffer from organ dysfunction, endothelial cell dysfunction, immune dysfunction and coagulation dysfunction, which will cause extremely serious physical damage to patients in a short time, or even lead to death. Therefore, we must find the most effective method to treat sepsis. Vitamin $\mathrm{C}$ is a polyhydroxy compound, which is very beneficial to human body and can help maintain vascular endothelial function and human immune function ${ }^{[2]}$. In this paper, 44 patients with sepsis treated in our hospital from June 2019 to June 2021 were investigated in order to analyze the effect and application value of vitamin $\mathrm{C}$ in the treatment of sepsis. The details are reported as follows.

\section{Materials and methods}

\subsection{General information}

A total of 44 patients with sepsis who were treated in our hospital from June 2019 to June 2021 were investigated and studied. The subjects were divided into conventional group and research group, with 22 patients in each group, by drawing lot methods. There were 12 males and 10 females in the conventional group, with an age range of 23-80 years and mean age of 49.34 \pm 4.20 years. There were 9 males and 13 females in the research group, with an age range of 25-73 years and mean age of 47.12 \pm 2.68 years. Critically ill patients were not included in this study. There was no significant difference in gender, age and 
other related data between the conventional group and the research group $(\mathrm{P}>0.05)$.

\subsection{Methods}

Routine methods, such as fluid resuscitation, use of antibiotics, removal of infection sources and use of vasoactive drugs, were used to treat sepsis in the patients of conventional group ${ }^{[3]}$. On the basis of these treatments, the the intake of vitamin $\mathrm{C}$ was increased in patients of the research group. Specifically, about $150 \mathrm{mg} / \mathrm{kg}$ of vitamin $\mathrm{C}$ was injected at a uniform rate within 5 hours every day by intravenous pumping ${ }^{[4]}$. The treatment time lasted for one week. The indexes of patients with sepsis were detected and recorded every 24 hours. After the treatment, the data are summarized, analyzed and processed, and finally the research results are compared.

\subsection{Evaluation criteria}

The effective rate of the two groups of sepsis patients after treatment were compared. The effective rate was categorized as follows: (1) Obviously effective: The patient's condition basically returned to normal and the physical infection basically disappeared; (2) Effective: The patient's condition was effectively controlled and the infection condition was improved; (3) Ineffective: The patient's condition did not change or worsened or he/she even died. The total effective rate is the ratio of the total number of obviously effective and effective persons to the total number of persons. The higher the value, the better the treatment effect.

The scores of liver function recovery in the two groups at different times were compared. There are two main indicators: (1) Alanine transaminase (ALT) which exists in human cells, especially hepatocytes. The higher the concentration of ALT, the slower the recovery of liver function; (2) Total bilirubin (TBIL) which is metabolized by the liver. The higher the concentration of TBIL, the slower the recovery of liver function ${ }^{[5]}$.

The scores of renal function recovery in the two groups at different times were compared. There are two main indicators: (1) Creatinine which is the product of human metabolism and needs to be excreted through the glomerulus. The higher the concentration of creatinine, the slower the recovery of renal function; (2) Uric acid which is also a product of human metabolism and exists in human body fluids. The higher the concentration of uric acid, the slower the recovery of renal function in patients with sepsis ${ }^{[6]}$.

\subsection{Statistical analysis}

SPSS version 24.0 statistical software was used to process the data. The count data are expressed as count (percentage), and were analyzed using $\chi^{2}$ test. The measurement data are expressed as mean \pm standard deviation, and were analyzed using $t$ test. Research results with $\mathrm{P}<0.05$ is statistically significant.

\section{Results}

\subsection{Comparison of therapeutic effects between the two groups of patients with sepsis}

The study found that the total effective rate of the research group $(95.45 \%)$ treated with vitamin $\mathrm{C}$ on the basis of conventional treatment was significantly higher than that of the conventional group $(72.72 \%)$ in which only conventional treatment was used $(\mathrm{P}<0.05)$, as shown in Table 1.

\subsection{Comparison of liver function recovery scores between the two groups in different time}

The ALT concentration and TBIL concentration in the research group treated with vitamin $\mathrm{C}$ were lower than those in the conventional group only using conventional treatment, indicating that the recovery of liver function in the research group was better than that in the conventional group $(\mathrm{P}<0.05)$, as shown in Table 2. 


\subsection{Comparison of renal function recovery scores between the two groups in different time}

The scores of creatinine and uric acid concentration in the research group treated with vitamin $\mathrm{C}$ were lower than those in the conventional group only using conventional treatment, indicating that the recovery of renal function in the research group was better than that in the conventional group $(\mathrm{P}<0.05)$, as shown in Table 3.

Table 1. Comparison of therapeutic effects between the two groups of patients with sepsis

\begin{tabular}{lcccc}
\hline Group & Obviously effective & Effective & Ineffective & Effective rate \\
\hline Research group $(\mathrm{n}=22)$ & $18(81.81)$ & $3(13.63)$ & $1(4.54)$ & $21(95.45)$ \\
Conventional group $(\mathrm{n}=22)$ & $9(40.90)$ & $7(31.81)$ & $6(27.27)$ & $16(72.72)$ \\
$\chi^{2}$ & & & & 4.2471 \\
$\mathrm{P}$ & & & & 0.0393 \\
\hline
\end{tabular}

Table 2. Comparison of liver function recovery scores between the two groups in different time

\begin{tabular}{lccccc}
\hline \multirow{2}{*}{ Group } & \multicolumn{2}{c}{ ALT $(\mathbf{U} / \mathbf{L})$} & & \multicolumn{2}{c}{ TBIL $(\boldsymbol{\mu m o l} / \mathbf{L})$} \\
\cline { 2 - 3 } \cline { 5 - 6 } & $\mathbf{2 4 h}$ & $\mathbf{7 2 h}$ & & $\mathbf{2 4 h}$ & $\mathbf{7 2 h}$ \\
\hline Research group $(\mathrm{n}=22)$ & $20.28 \pm 10.02$ & $18.26 \pm 9.57$ & & $11.12 \pm 3.58$ & $10.26 \pm 3.49$ \\
Conventional group $(\mathrm{n}=22)$ & $31.34 \pm 21.17$ & $31.65 \pm 25.04$ & & $15.72 \pm 9.37$ & $14.88 \pm 7.85$ \\
$\mathrm{t}$ & 2.2149 & 2.3429 & & 2.1510 & 2.5224 \\
$\mathrm{P}$ & 0.0322 & 0.0239 & & 0.0373 & 0.0155 \\
\hline
\end{tabular}

Table 3. Comparison of renal function recovery scores between the two groups in different time

\begin{tabular}{lccccc}
\hline \multirow{2}{*}{ Group } & \multicolumn{2}{c}{ Creatinine $(\boldsymbol{\mu m o l} / \mathbf{L})$} & & \multicolumn{2}{c}{ Uric acid $(\boldsymbol{\mu m o l} / \mathbf{L})$} \\
\cline { 2 - 3 } \cline { 5 - 6 } & $\mathbf{2 4 h}$ & $\mathbf{7 2 h}$ & & $\mathbf{2 4 h}$ & $\mathbf{7 2 h}$ \\
\hline Research group $(\mathrm{n}=22)$ & $64.43 \pm 30.28$ & $56.05 \pm 24.13$ & & $174.95 \pm 97.78$ & $153.42 \pm 75.29$ \\
Conventional group $(\mathrm{n}=22)$ & $92.71 \pm 47.56$ & $90.21 \pm 65.57$ & & $252.14 \pm 145.68$ & $235.28 \pm 172.79$ \\
$\mathrm{t}$ & 2.3526 & 2.2932 & & 2.0635 & 2.0371 \\
$\mathrm{P}$ & 0.0234 & 0.0269 & & 0.0453 & 0.480 \\
\hline
\end{tabular}

\section{Discussion}

Sepsis has gradually evolved into a severe disease with a higher death rate than critical heart diseases. Once patients suffer from sepsis, their health and life will be severely affected. Sepsis is mainly caused by uncontrolled inflammatory reaction, which is caused by various types of infections, including lung infection, biliary tract infection, abdominal infection, urinary system infection, intracranial infection and systemic soft tissue infection. In addition, a patient with other critical diseases is prone to develop infection, which eventually develops into sepsis. These critical diseases include severe burns, diabetes, leukemia, chronic obstructive bronchial disease, aplastic anemia and immune system diseases ${ }^{[7]}$. Sepsis will lead to insufficient perfusion of organs in patients, resulting in organ dysfunction, and death in more serious cases. Therefore, we must find the most effective method to treat sepsis. The conventional treatment methods for sepsis are mainly infection control, fluid resuscitation and organ support therapy, but the therapeutic effect for sepsis with high mortality needs to be improved

Vitamin $\mathrm{C}$ can effectively maintain the function of endothelial cells and improve the immunity, so as to maintain the stability of organ function of patients. It is found that after vitamin $\mathrm{C}$ treatment, the treatment 
efficiency of patients is greatly improved, the concentrations of ALT and TBIL in human body are decreased, the liver function of patients is effectively improved, the concentrations of creatinine and uric acid are also decreased, and the renal function of patients was gradually returning to normal. In view of this, vitamin $\mathrm{C}$ can effectively improve the organ function damaged by sepsis in patients, and fundamentally provide effective treatment for patients with sepsis. This study uses vitamin $C$ to treat patients with sepsis on the basis of conventional treatment methods. By observation and comparison, the total effective rate of research group (95.45\%) was much higher than that of the conventional group (72.72\%), and the scores of liver function and renal function in the research group were much higher than those in the conventional group at different times $(\mathrm{P}<0.05)$.

In view of this, vitamin $\mathrm{C}$ has a good effect in the treatment of sepsis. It not only greatly reduces the mortality of sepsis patients, but also effectively improves the liver function and renal function of sepsis patients. Thus, vitamin $\mathrm{C}$ is of high application value and should be popularized for applications.

\section{Disclosure statement}

The author declares no conflict of interest.

\section{References}

[1] Li XY, Li PJ, 2019, Vitamin C: The Choice of Adjuvant Therapy for Sepsis. Chinese Journal of Critical Care Medicine, 39(11): 1110-1114.

[2] Chang XN, Li M, Zhang ZX, et al., 2019, Meta-Analysis of the Efficacy of Vitamin C in the Treatment of Sepsis and Septic Shock. Chinese Journal of Critical Care Medicine (Electronic Edition), 12(1): 37 41.

[3] Zhao RR, Li SW, Wan XY, 2021, Research Progress on the Effect of Vitamin C on Microcirculation in Sepsis. Chinese Journal of Internal Medicine, 60(9): 852-856.

[4] Gao WP, Luo Y, Zhu JW, 2020, Research Progress on Vitamin C in the Treatment of Sepsis. Clinical Medicine, 40(11): 123-124, post insertion 1 - post insertion 2.

[5] Chen C, Zhang JZ, Wan XY, 2019, Application of High-dose Vitamin C in Sepsis. Chinese Journal of Internal Medicine, 58(3): 233-236.

[6] Lv QQ, Gu XH, Lv YM, et al., 2020, Application Progress of Vitamin C in Sepsis. Chinese Journal of Critical Care \& Intensive Care Medicine (Electronic Edition), 6(1): 104-108.

[7] Cai Y, 2020, Therapeutic Effect of High-dose Vitamin C on Myocardial Injury in Patients with Sepsis and Its Possible Mechanism. Journal of Clinical Medical Literature, 7(95): 124, 126. 